\title{
Immediately Sequential Bilateral Cataract Surgery-A Global Perspective
}

\author{
Steve A Arshinoff, MD, FRCSC \\ Directing Partner, York Finch Eye Associates, Humber River Hospital, Toronto, Ontario, Canada; Assistant Professor, Department of Ophthalmology and Vision Sciences, \\ University of Toronto, Toronto, Ontario, Canada; Assistant Clinical Professor, Surgery (Adjunct), McMaster University, Hamilton, Ontario, Canada
}

\begin{abstract}
By the mid 1990s, the evolution of progressively safe and predictable cataract surgery saw the resurgence of centuries of interest in performing immediately sequential bilateral cataract surgery (ISBCS). Within 10 years ISBCS had become generally accepted and the International Society of Bilateral Cataract Surgeons (iSBCS) was formed (September 2008) to "promote education, mutual cooperation, and progress in simultaneous bilateral cataract surgery." The first initiative of the society members was to create a document "iSBCS General Principles for Excellence in ISBCS 2009," which was intended to disseminate information about the best practices they had discovered to assist novice ISBCS surgeons. Next, ISBCS needed to be studied. Soon, data began to clarify some advantages of ISBCS, and risks were carefully evaluated. It soon became apparent that the main impediment to the performance of ISBCS, globally, was money, in that many jurisdictions financially penalized surgeons who performed ISBCS. Presently, we know that ISBCS carries many benefits to the patient, his/her family, the surgical facility, and society. The feared risks for simultaneous bilateral endophthalmitis, the requirement to adjust intraocular lens (IOL) selection for second eyes, based on first eye results, and others, have simply not been borne out under scrutiny. ISBCS is now rapidly increasing in its performance and acceptance globally, but the financial factors remain to be solved.
\end{abstract}

\section{Keywords}

Bilateral cataract surgery, immediately sequential bilateral cataract surgery, cataract surgery, endophthalmitis, simultaneous bilateral endophthalmitis

Disclosure: Steve A Arshinoff, MD, FRCSC, has no conflicts of interest to declare. No funding was received in the publication of this article.

Open Access: This article is published under the Creative Commons Attribution Noncommercial License, which permits any noncommercial use, distribution, adaptation, and reproduction provided the original author(s) and source are given appropriate credit.

Received: January 12, 2015 Accepted: February 9, 2015 Citation: US Ophthalmic Review, 2015;8(1):14-8 DOI: 10.17925/usor.2015.8.1.14

Correspondence: Steve A Arshinoff MD, FRCSC, York Finch Eye Associates, 2115 Finch Ave W \#316, Toronto, Ontario, Canada M3N 2V6. E: ifix32is@gmail.com

"The argument of whether to operate bilateral cataracts in a single session, or in two different sessions, is as old as the operation itself, and the controversy between supporters of one option and the other had already, in the Middle Ages, become fiercely heated. So it has stayed, over the centuries, up to the present day."1 Few suggestions of how to advance cataract surgery have given rise to as much heated discussion, often without scientific backing for claims and accusations. Taking a broader perspective, Robert Wright has written eloquently on how everything tends to amalgamations and increasing complexity, as long as the parties engaged in the effort experience a nonzero sum of benefits. ${ }^{2}$ It should follow from this that the ultimate acceptance of bilateral cataract surgery is inevitable.

I have been practicing fairly routine immediately sequential bilateral cataract surgery (ISBCS) since 1996, when a 35-year-old, female, racing car driver demanded that I perform her two cataract surgeries at the same sitting. She informed me of her tight schedule, and stated that she had researched cataract surgery and surgeons carefully, and concluded that the risk was far lower than that of her usual occupation. She was prepared to sign whatever consent I wanted to give her. Her result was excellent and her joy at recovering bilateral excellent vision in one day so dramatic that I decided to offer ISBCS to selected patients, and soon after to all patients. To date I have performed over 9,000 phacoemulsification procedures as ISBCS, and have never encountered a complication that was a result of the bilaterality of the procedure (such as bilateral postoperative endophthalmitis, corneal edema, etc.) I was, however, concerned about any possible increased risk for the procedure compared with delayed sequential bilateral cataract surgeries (DSBCS), and so I adopted a number of strict practices, which I believe to be essential to the safe practice of ISBCS:

1. Intracameral antibiotics are used in every case.

2. Complete sterile separation of the two procedures is critical, with reprepping and redraping between eyes, the use of different sets of instruments and different lots of balanced salt solution (BSS), disposables, and ophthalmic viscosurgical devices (OVDS).

3. If any unmanaged complication occurs with the first eye, the second eye is deferred. (This has been an extremely rare occurrenceperhaps five in nearly 20 years, and most of those due to nonocular 
issues: patient complaint of sore back, our instructions to the patient not having been properly translated to a non-English speaking patient by the family, etc.)

4. Information of right and left eye intraocular lens (IOL) and astigmatism clearly marked on a board in the operating room (OR) for all staff to see. OR nurses are trained to read and interpret biometric data, which they read out loud as they pass the IOL from the circulating table to the scrub nurse, confirming IOL choice. We have had no IOL errors since adopting this policy.

Twelve years after I began to perform ISBCS, on September 1, 2008, the International Society of Bilateral Cataract Surgeons (iSBCS, available at www.isbcs.org) was set up, with me as founding president. The organization was established to share experiences and input on best practices from many different practitioners from many countries. We hoped to formulate principles of safe practice, and suggestions for newcomers to ISBCS based on broad experience and evidence. I will refer to some of the views and rules of "the society" rather than my own, because they have been extensively discussed and reviewed by a well-informed global group. Our first task was to generate from the experiences of the members a document "iSBCS General Principles for Excellence in ISBCS 2009," which was finalized after 1 year, in September 2009, published on our website, and appended to this article (see Appendix).

Our society has one ISBCS rule: "If any unresolved complication occurs with the first eye, the second eye should be deferred." However, every member will attest: "The best time to operate the second eye is immediately after gaining the experience of the individual peculiarities of the first eye, and the second eye is always easier for both the doctor and the patient."

\section{Advantages of ISBCS}

\section{Avoiding Fear of Surgery for a Patient who} Experienced a Problem with their First Eye Surgery Most ophthalmic surgeons have a few patients who experienced a disastrous complication with their first cataract surgery. As our techniques gradually advance, this is becoming less common, but I have had two patients who came to me with such terrible surgical phobia, as a consequence of bad experiences, that they are now functionally blind with an operable cataract in their only eye, but would rather have guiding vision than submit to surgery, no matter what I say, or whose second opinion I obtain for them. It is a tragedy that never occurs with ISBCS.

\section{Greater Visual Improvement After Second Eye Surgery than After First Eye Surgery}

In the 1990s, ophthalmologists were challenged about why it was or was not necessary to perform cataract surgery on second eyes. Numerous studies, the most prominent of which were by Javitt 1995, Swedish outcomes study 1997, and the Royal College of Ophthalmologists 2001, all confirmed that there is more functional visual improvement after second eye surgery than after first eye surgery. This makes perfect sense. Single eye surgery causes a reduction of our visual system from two receptors to one. There are no surviving species with single eyes, so it appears that natural selection also decided that two eyes are better than one. Second eye surgery restores a normal balanced visual system for the patient, something that nature has validated for millions of years.
Figure 1: The First Academic Meeting of the International Society of Bilateral Cataract Surgeons

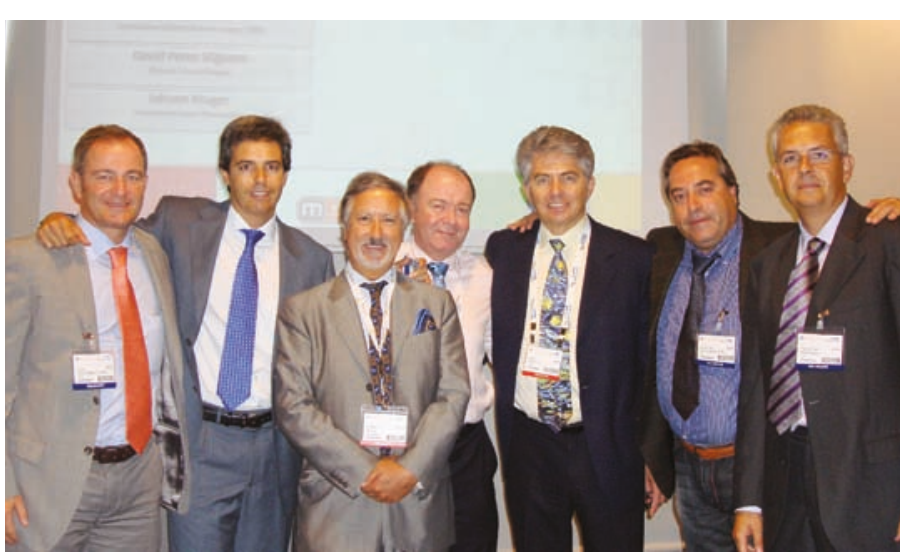

At this meeting, held on September 14, 2009, in Barcelona, Spain, the "iSBCS General Principles for Excellence in ISBCS 2009" were agreed upon and accepted. Left to right: Javier Goas Iglesias de Ussel, David Pérez Silguero, Charles Claoué, John Bolger, Steve Arshinoff, Ramon Henriques de la Fé, and Miguel Ángel Pérez Silguero. Absent from photo: Björn Johansson, Johann Kruger, Ilan Sebban, Jonathan Christenbury, Richard Packard, Manuel Diaz Llopis, David Lane, Christian Ferremi, Erik Mertens, Roberto Gallego-Pinazo, Clive Peckar, Pierre Demers, and Tejas Shah.

\section{Immediate Rehabilitation of the Visual System}

Unilateral cataract surgery repairs one peripheral receptor, whereas ISBCS rehabilitates the visual system, with much more rapid return to full visual function. Lundstrom et al. demonstrated, in 2006, that what ISBCS achieves almost immediately post-op, separate procedures can accomplish only up to 4 months after the second eye surgery. ${ }^{3}$

\section{Better Planning of the Refractive Result, and Treatment for Anismetropic Amblyopia}

There has been a lot written in the past decade about correcting biometry for the second eye based upon the refractive result in the first eye. The initial paper by Jabbour et al. ${ }^{4}$ found no benefit, but then a few papers appeared that seemed to show a small benefit. The best study by far, and the one that summarizes the entire situation, is that of Thomas Olsen who demonstrated that the benefit of adjusting biometric calculation error for the second eye based upon the result from the first eye is inversely proportional to the quality of the biometry used, with negligible benefit with the Lenstar and the Olsen formula. ${ }^{5}$ In other words: the more accurate the measurements and the chosen formula, the less the benefit of adjustment, until it becomes vanishingly small with modern biometric techniques.

Additionally ISBCS permits the surgeon to plan whatever refraction the patient wants postoperatively, whether it be plano, myopia, monovision, multifocal IOLS, etc., regardless of the patient's preoperative refraction, without concern about induction of significant bothersome anisometropia until the second eye is completed. This is a huge benefit when dealing with high preoperative hyperopia, astigmatism, or myopia.

Furthermore, ISBCS allows the surgeon to operate on refractive amblyopic eyes, as sort of a bonus procedure for the patient who presents for unilateral surgery on their "good eye," when surgery on the amblyopic forgotten eye might otherwise never have been carried out. I have performed an increasing number of these cases, often with remarkable and surprisingly good results. Many -15 to -25 D myopic eyes that had mild cataracts and acuity less than 20/200 achieved better 
than 20/40 vision post-op, and many anisometropic hyperopic eyes with and without significant astigmatism had been given up for years, but achieved acuities of 20/30 to 20/60, when they were barely $20 / 200$, or worse preoperatively. And some of them continue to improve by a few lines of acuity for a few years.

\section{Fewer Patient Visits}

Clearly, fewer patient visits to the ophthalmologist are needed when one operative episode occurs rather than two. The decrease in the total number of visits is generally three, unless the two procedures are performed in very close proximity (which begs the question of why ISBCS was not carried out, because performing very close DSBCS averts little risk for ISBCS, but loses most of the benefit). Bolger has shown mathematically that the risk for bilateral endophthalmitis with ISBCS is less than the risk for dying in a traffic accident driving back and forth for the extra visits required by DSBCS. ${ }^{6}$

\section{The Added Risks for ISBCS}

ISBCS has not been demonstrated to have any increased risk compared with DSBCS.7.8 The major concern expressed has been the purported increased risk for unilateral or bilateral postoperative endophthalmitis. The multicentred study of endophthalmitis after cataract surgery performed by Arshinoff and Bastianelli with iSBCS members revealed the lowest post-operative infection rates ever reported $(1: 17,000)$, and zero bilateral infections in over 100,000 eyes. ${ }^{9}$ Li et al. reported that the risk for simultaneous bilateral endophthalmitis (SBE) after ISBCS is less than the risk for death after general anesthesia. ${ }^{10}$ There have been four cases of SBE reported, all with significant breaches of modern sterile protocol.

Other risks cited as reasons not to perform ISBCS include the risk for bilateral postoperative retinal detachment, corneal edema, or macular edema, particularly in people with diabetes. Although there is no reason to expect that any of these risks are increased in an individual eye, as a consequence of bilateral surgery, judgment is required in selecting patients for ISBCS, and patients at high risk for the above represent relative contraindications, or cases in which special precautions may be needed (pre and postoperative retinal assessments, endothelial cell counts, etc.)

\section{Money and ISBCS}

Although it is often an unspoken concern, the greatest problem for the acceptance of ISBCS globally is money. For unknown reasons, the majority of jurisdictions globally financially penalize ISBCS. This is completely nonsensical, as ISBCS has been shown to save money for everybody, and penalizing it simply results in decreasing its popularity, and thereby costing everybody concerned much more than cataract surgery should. Finland has never penalized bilateral surgery, and so it is the country that performs ISBCS most commonly: about half of all patients undergo ISBCS. Leivo et al. have studied the differential in costs between unilateral and bilateral cataract surgery in Finland, and estimated the ISBCS savings at $€ 1,600(\$ 1,827)$ per patient. Then, in a stroke of brilliance, the Leivo group decided to estimate how much extra a country would spend by performing all cataracts as DSBCS rather than ISBCS per SBE theoretically prevented by doing so, believing that DSBCS prevents bilateral simultaneous infections. They took the estimated infection rate at 1:1000, and therefore estimated the SBE rate at $(1: 1,000)^{2}$. From this they calculated that the cost to prevent SBE would be
$€ 739$ million, or about $\$ 1$ billion per SBE case prevented. ${ }^{11}$ It is important to note that this does not prevent bilateral infection at that rate, which remains a mathematical risk, it only prevents the two infections from being simultaneous. O'Brien et al. repeated this study using Canadian data, and arrived at a very similar CAD $\$ 2,000$ saving per ISBCS patient. If this result is combined with the more recently calculated current global declining risk for endophthalmitis found among isBCS bilateral surgeons by Arshinoff and Bastianelli (1:17,000 when intracameral antibiotics are used), and squared using Leivo's method, the risk for SBE comes out to 1:289 million, and the additional cost incurred by performing only DSBCS per single hypothetical case of SBE prevented exceeds US $\$ 250$ billion. This incredible amount of money exceeds the health budget of most countries, and would never be approved by any country for any treatment of a single patient, or even a large group of patients.

ISBCS saves money and time for everyone concerned: the patient, the family of the patient, the doctor, the treating institution, and the health care system. Patients and families love ISBCS and after surgery send in their friends. It would seem wise for health jurisdictions to simply pay the same for each eye whether performed as DSBCS or ISBCS. The effect of this policy would be that the payers in the system would be voluntarily sharing some of the savings they garner from ISBCS with surgeons, patients, and surgical centers. Governments endlessly deliberate how to distribute their finances, fully aware that the choices they make change behavior in society. They are foolishly preventing ISBCS in most jurisdictions and wasting huge amounts of taxpayers' money for no benefit. A decision to change and share the benefit of ISBCS would help everybody.

\section{Conclusion}

ISBCS is becoming increasingly popular globally, as it has been found to have numerous benefits and few drawbacks. Although there are clearly instances when DSBCS is generally preferred (Fuchs' endothelial dystrophy or other cause of bilateral low endothelial cell counts, diabetic maculopathy, subluxated or luxated cataracts, etc.), as a surgeon gains experience with ISBCS the number of absolute exclusions decreases, and iSBCS surgeons believe that the easiest time to perform surgery on an eye is immediately after gaining the experience of the surgical idiosyncrasies of the other eye. So, in anticipated complex cases, these two factors must be carefully balanced. A problem arises in femtosecond cases where it is desirable to perform the femto part of the surgery on both eyes before performing the phaco on the first eye. If a problem occurs during phaco with the first eye, in this circumstance, it is too late to defer the second eye surgery. This issue requires more experience and study before femtofemto-phaco-phaco procedures become accepted.

But the main disincentive to the performance of ISBCS is really financial in all investigated jurisdictions where it is unpopular. In the US, ISBCS is popular in patient-pay refractive cataract surgery, but unpopular in feefor-service cataract surgery, as American surgeons and their surgery centers are penalized $50 \%$ of their fees by Medicare for surgery on the second eye on the same day as the first eye. Health maintenance organizations, however, gain financially from ISBCS, and are beginning to encourage it. Stiverson reported at the 2014 American Society of Cataract and Refractive Surgery (ASCRS) meeting that $80 \%$ of patients who are candidates for ISBCS, voluntarily choose it over DSBCS, when allowed to choose freely. ${ }^{12}$ This is almost exactly the same percentage I experience 
in Canada. The greatest number of new members to iSBCS in 2014 came from the US. This was a sharp difference from preceding years, when few members were Americans (in proportion to population size), indicating a large recent rise in interest in ISBCS in the US. iSBCS now has members from Argentina, Australia, Austria, Belgium, China, Colombia, Cyprus, Czech Republic, Denmark, Estonia, France, Germany, Greece, Hong Kong, Hungary, India, Iran, Italy, Japan, Korea, Kuwait, Malaysia, Mexico, New
Zealand, Norway, Philippines, Poland, Portugal, Romania, Russia, Serbia, South Africa, Slovenia, Switzerland, Syria, Turkey, and Ukraine. The growth rate has accelerated in the past 2 years.

I expect that I will surpass my ISBCS eye number 10,000 and 20 years of performing routine ISBCS before ISBCS becomes fully accepted and common in most of the world, but not by a very long period of time.
1. del Castillo M, Operación de las cataratas bilaterales en sesión única, Studium Ophthalmologicum, 1986;5:84-8. (Translated by David Perez Silguero, MD, Gran Canaria, Spain).

2. Wright R, Nonzero: The Logic of Human Destiny, New York: Vintage Books, 2001.

3. Lundström M, Albrecht S, Nilsson, Åström B, Benefit to patients of bilateral same-day cataract extraction: Randomized clinical study, J Cataract Refract Surg, 2006;32:826-30.

4. Jabbour J, Irwig L, Macaskill P, et al., Intraocular lens power in bilateral cataract surgery: Whether adjusting for error of predicted refraction in first eye improves prediction in the second eye, I Cataract Refract Surg, 2006;32:2091-7.

5. Olsen T, Use of fellow eye data in the calculation of intraocular lens power for the second eye, Ophthalmology, 2011:118:1710-5.

6. Ellis MF, Bolger J, Steinmetz B, Claoué C, Responses to December 1997 consultation section [letters], J Cataract Refract Surg, 1998;24:430-2.

7. Arshinoff SA, Strube Yi, Ning J, Yagev R, Simultaneous bilateral cataract surgery, J Cataract Refract Surg, 2003;29:1281-91.

8. Serrano Aguilar P, Seguridad, efectividad y costeefectividad de la cirugía de cataratas bilateral y simultánea frente a la cirugia bilateral de cataratas en dos tiempos, Santa Cruz de Tenerife: Servicio Canario de la Salud.

9. Arshinoff SA, Bastianelli PA, Incidence of postoperative endophthalmitis after immediately sequential bilateral cataract surgery (ISBCS), JCRS, 2011:37:2105-114.

10. Li O, Kapetanakis V, Claoué C, Simultaneous bilateral endophthalmitis after immediate sequential bilateral cataract surgery: What's the risk of functional blindness, Am J Ophth, 2014;158:749-50

11. Leivo T, Sarikkola AU, Uusitalo RJ, et al., Simultaneous bilatera cataract surgery: Economic analysis: Helsinki Simultaneous Bilateral Cataract Surgery Study Report 2, J Cataract Refract Surg, 2011;37:1003-8.

12. Stiverson $\mathrm{K}$, Kloor J, Litoff $\mathrm{D}$, Bilateral cataract surgery in the Colorado Permanente Medical Group. Presented at the 2014 American Society of cataract and Refractive Surgery meeting by Kent Stiverson, Boston, Massachussets, April 27, 2014. 